\title{
Preface to Topical Issue: Waves in the Solar Corona: From Microphysics to Macrophysics
}

\author{
V.M. Nakariakov ${ }^{1,2}$ • D.J. Pascoe ${ }^{1} \cdot$ R. Sych ${ }^{3}$. \\ L. van Driel-Gesztelyi ${ }^{4,5,6}$
}

Published online: 21 November 2016

(C) Springer Science+Business Media Dordrecht 2016

Wave and oscillatory processes in the plasma of the solar corona attract growing attention in the context of key challenges in solar physics. For several decades, waves have been considered as one of the mechanisms for coronal plasma heating (e.g. De Moortel and Browning, 2015) and solar wind acceleration (e.g. Cranmer, 2012). Coronal waves are intensely used for plasma diagnostics, from radio emission to large-scale magnetohydrodynamic (MHD) timescales (see, e.g. Nakariakov, Kashapova, and Yan, 2014; Liu and Ofman, 2014). The waves are natural agents that can establish the connectivity of different layers of the solar atmosphere (Komm et al., 2015). Interesting perspectives have recently been identified for the deep exploitation of the coronal-magnetospheric analogy (Nakariakov et al., 2016). This shows that the study of solar coronal waves and oscillations is an active and rapidly developing research topic. This Topical Issue addresses the recent achievements in the field, and contains two invited review papers and eighteen original papers, covering new interesting observational and theoretical results.

The invited review of Van Doorsselaere, Kupriyanova, and Yuan (2016) discusses the recent progress in the observational detection and modelling of quasi-periodic pulsations

Waves in the Solar Corona: From Microphysics to Macrophysics

Guest Editors: Valery M. Nakariakov, David J. Pascoe, and Robert A. Sych

V.M. Nakariakov

V.Nakariakov@ warwick.ac.uk

1 Centre for Fusion, Space and Astrophysics, Department of Physics, University of Warwick, Coventry CV4 7AL, UK

2 School of Space Research, Kyung Hee University, Yongin, 446-701, Gyeonggi, Korea

3 Institute of Solar-Terrestrial Physics of Siberian Branch of Russian Academy of Sciences, Lermontov St. 126a, PO Box 291, Irkutsk, 664033 Russia

4 Mullard Space Science Laboratory, University College London, Holmbury St. Mary, Dorking, Surrey, RH5 6NT, UK

5 LESIA, Observatoire de Paris, 5 place Jules Janssen, 92195 Meudon, France

6 Konkoly Observatory, Budapest, Hungary 
(QPP) of the electromagnetic emission generated by solar and stellar flares, which have recently been shown to be an inherent feature of flares. The potential of QPP for plasma diagnostics is addressed specifically.

The invited review of Mishin and Tomozov (2016) emphasises the analogy between the wave-flow interactions that occur in the solar corona and wind, and in the Earth's magnetosphere. The exploitation of this analogy opens up interesting perspectives for the crossfertilisation of these two research fields.

Afanasyev and Uralov (2016) investigate with the use of the ray-tracing method how slow magnetoacoustic waves penetrate the flare reconnection site. By transforming into a fast wave, the wave can produce a periodic modulation of the physical conditions in the vicinity of a magnetic null point, which in turn produces QPP. A similar effect was studied by Chen et al. (2016), who present a numerical simulation of a fast magnetoacoustic wave interacting with a magnetic quasi-separatrix layer. The conversion of the fast mode to a slow mode is found to form an apparently stationary wave front, similar to what has been detected in observations. Smirnova et al. (2016) perform two-dimensional (2D) numerical simulations of an impulsive pressure perturbation interacting with the null point of a potential magnetic arcade. The formation of double-structured jets comprised of a cold and dense lower region and a hotter and less dense upper region resembles properties of type I and type II spicules, respectively, that have been observed in the solar atmosphere. The study of the association between the chromospheric Moreton wave and the coronal extreme ultraviolet (EUV) signatures with the Atmospheric Imaging Assembly onboard the Solar Dynamics Observatory (SDO/AIA) shows that Moreton wave fronts can be produced by a coronal shock-wave that pushes the chromosphere downward (Francile et al., 2016). A 3D geometrical model that explains the observed dynamics of the wave-front structure is proposed. The results of the model are found to be in agreement with the coronal shock-wave generated by a 3D piston that can be attributed to the coronal mass ejection (CME) front.

Anfinogentov and Nakariakov (2016) develop a novel technique for the detection of quasi-periodic displacements in imaging data cubes, in particular, kink oscillations of coronal loops and filaments. Their motion magnification allows the study of low-amplitude (subresolution) oscillations. The authors tested this method on synthetic data and data obtained with SDO/AIA and demonstrated its robustness. Sarkar et al. (2016) studies large-amplitude and long-period (about $30 \mathrm{~min}$ ) transverse oscillations in a coronal loop that are excited by a jet. The estimation of the magnetic field inside the oscillating loop, made with the seismological inversion technique, shows that the energy of the jet is greater than the energy density of the oscillation, which supports this interpretation. Standing, rapidly decaying waves of EUV intensity, with periods of $9 \mathrm{~min}$ and $19 \mathrm{~min}$, are detected in a coronal active region by Conde Cuellar, Costa, and Cedeño Montaña (2016). The asymmetry in the magnetic field along the oscillating loop was shown to affect the properties of these oscillations. Pant et al. (2016) report a rare simultaneous detection of longitudinal and transverse oscillations in a filament following a solar flare using data from GONG H $\alpha$ and SDO/AIA $171 \AA$ A. The oscillation period is used to obtain a seismological estimate for a lower limit of the magnetic field strength of $25 \mathrm{G}$.

The magnetic field structure of the solar atmosphere, determined with the use of multiwavelength observations of the polarised radio emission with RATAN-600 is obtained by Bogod and Yasnov (2016). Two methods are used: a stereoscopic method and the analysis of the radio spectrum. The obtained results show high values of the magnetic field strength with height, which indicates a weak divergence of the magnetic flux tubes in the active regions, while some polarised radio sources revealed a spiral magnetic structure. 
Multi-modal oscillations in facular magnetic knots are studied at different layers of the solar atmosphere by Chelpanov, Kobanov, and Kolobov (2016). The properties of these oscillations are probably determined by the magnetic field configuration and hence the cut-off frequency. At low heights, the magnetic field is close to vertical and does not significantly affect the oscillations. However, with increasing latitude, the field lines are inclined, which enables low-frequency waves to propagate upward and reach the corona and, in particular, flaring sites. Kolobov, Chelpanov, and Kobanov (2016) study the spatial distributions of the spectral power of sunspot oscillations, using emission lines that correspond to different atmospheric heights to demonstrate the existence of consistent structures. While the higher frequency oscillations are localised in the umbra, the lower frequency oscillations are found to be associated with the surrounding inclined magnetic field lines, providing support for the hypothesis that similar physical conditions extend from the chromosphere to the transition region. Tsap, Stepanov, and Kopylova (2016) analyse theoretically the phase relationships between the disturbed quantities of evanescent acoustic and slow sausage modes of photospheric magnetic waveguides. These relationships are found to depend on the frequency, and to agree well with the phase relationships observed with Hinode/SOT.

Efremov, Parfinenko, and Solov'ev (2016) use data from the Global Oscillation Network Group to study an ultra low-frequency oscillation of a chromospheric filament on the solar disk. Using a five-day $\mathrm{H} \alpha$ time series, they reveal oscillations with a period of $20-30$ hours. Different segments of the filament are found to oscillate with different periods.

Gao et al. (2016) and co-authors provide an explanation of the broken lane in type II radio bursts, interpreting it in terms of the collision of a coronal shock with the flare-CME current sheet. The thickness of the current sheet is estimated by a lane gap, and the results agree well with previous estimations.

Kuznetsov et al. (2016) analyse the spatio-temporal evolution of hard X-ray QPPs, revealing that the sources of the pulsations are not stationary, but move within the flaring active region. It is suggested that the apparent motion is associated with the successive triggering of energy release in different loops by an erupting magnetic rope. Kupriyanova et al. (2016) detected 40-50 s QPP of the microwave, X-ray, and radio emissions associated with nonthermal electrons accelerated in a solar flare. This finding indicates that the QPPs are likely to be caused by the periodic injection of non-thermal electrons, which can be produced by periodic modulation of magnetic reconnection. Similar short-period QPPs of hard X-ray and gamma-ray emissions, with periods ranging from 5 to 9 seconds, are detected in about one third of all flares that were produced in six weeks in the same active region (Myagkova et al., 2016), suggesting that the magnetic configuration of the active region remained stable for the duration of the observations.

Zaitsev, Kronshtadtov, and Stepanov (2016) consider the electric fields generated by the Rayleigh-Taylor instability in the chromospheric legs of current-carrying magnetic loops, where high densities alleviate the problem of providing sufficient accelerated electrons that applies to coronal sources. The conditions required to generate super-Dreicer electric fields are determined and demonstrated to accelerate electrons and protons, which greatly increases the energy release in flaring emission.

This Topical Issue is partly based upon the activities of the Marie Curie International Research Staff Exchange network PIRSES-GA-2011-295272 "Radiophysics of the Sun" (2012 - 2016, PI V. M. Nakariakov), and the 4th RadioSun Workshop and Summer School held in Irkutsk, Russia from 8-12 June 2015. 


\section{References}

Afanasyev, A.N., Uralov, A.M.: 2016, Slow-mode MHD wave penetration into a coronal null point due to the mode transmission. Solar Phys. 291. DOI. ADS.

Anfinogentov, S., Nakariakov, V.M.: 2016, Motion magnification in coronal seismology. Solar Phys. 291. DOI.

Bogod, V.M., Yasnov, L.V.: 2016, Determination of the structure of the coronal magnetic field using microwave polarization measurements. Solar Phys. 291. DOI. ADS.

Chelpanov, A.A., Kobanov, N.I., Kolobov, D.Y.: 2016, Influence of the magnetic field on oscillation spectra in solar faculae. Solar Phys. 291. DOI. ADS.

Chen, P.F., Fang, C., Chandra, R., Srivastava, A.K.: 2016, Can a fast-mode EUV wave generate a stationary front? Solar Phys. 291. DOI. ADS.

Conde Cuellar, S.M., Costa, J.E.R., Cedeño Montaña, C.E.: 2016, Oscillations in the flaring active region NOAA 111272. Solar Phys. 291. DOI.

Cranmer, S.R.: 2012, Self-consistent models of the solar wind. Space Sci. Rev. 172, 145. DOI. ADS.

De Moortel, I., Browning, P.: 2015, Recent advances in coronal heating. Phil. Trans. Roy. Soc. London Ser. A, Math. Phys. Sci. 373, 20140269. DOI. ADS.

Efremov, V.I., Parfinenko, L.D., Solov'ev, A.A.: 2016, Ultra low-frequency oscillations of a solar filament observed by the GONG network. Solar Phys. 291. DOI.

Francile, C., López, F.M., Cremades, H., Mandrini, C.H., Luoni, M.L., Long, D.M.: 2016, Moreton and EUV waves associated with an X1.0 flare and CME ejection. Solar Phys. 291. DOI. ADS.

Gao, G., Wang, M., Wu, N., Lin, J., Ebenezer, E., Tan, B.: 2016, The broken lane of a type II radio burst caused by collision of a coronal shock with a flare current sheet. Solar Phys. 291. DOI.

Kolobov, D.Y., Chelpanov, A.A., Kobanov, N.I.: 2016, Peculiarity of the oscillation stratification in sunspot penumbrae. Solar Phys. 291. DOI. ADS.

Komm, R., De Moortel, I., Fan, Y., Ilonidis, S., Steiner, O.: 2015, Sub-photosphere to solar atmosphere connection. Space Sci. Rev. 196, 167. DOI. ADS.

Kupriyanova, E.G., Kashapova, L.K., Reid, H.A.S., Myagkova, I.N.: 2016, Relationship of type III radio bursts with quasi-periodic pulsations in a solar flare. Solar Phys. 291. DOI. ADS.

Kuznetsov, S.A., Zimovets, I.V., Morgachev, A.S., Struminsky, A.B.: 2016, Spatio-temporal dynamics of sources of hard X-ray pulsations in solar flares. Solar Phys. 291. DOI. ADS.

Liu, W., Ofman, L.: 2014, Advances in observing various coronal EUV waves in the SDO era and their seismological applications (Invited review). Solar Phys. 289, 3233. DOI. ADS.

Mishin, V.V., Tomozov, V.M.: 2016, Kelvin-Helmholtz instability in the solar atmosphere, solar wind and geomagnetosphere. Solar Phys. 291. DOI. ADS.

Myagkova, I.N., Bogomolov, A.V., Kashapova, L.K., Bogomolov, V.V., Svertilov, S.I., Panasyuk, M.I., Kuznetsova, E.A., Rozhkov, G.V.: 2016, Solar X-ray emission measured by the Vernov mission during September-October of 2014. Solar Phys. 291. DOI. ADS.

Nakariakov, V.M., Kashapova, L.K., Yan, Y.-H.: 2014, Editorial: solar radiophysics: recent results on observations and theories. Res. Astron. Astrophys. 14, 001. DOI. ADS.

Nakariakov, V.M., Pilipenko, V., Heilig, B., Jelínek, P., Karlický, M., Klimushkin, D.Y., Kolotkov, D.Y., Lee, D.-H., Nisticò, G., Van Doorsselaere, T., Verth, G., Zimovets, I.V.: 2016, Magnetohydrodynamic oscillations in the solar corona and Earth's magnetosphere: towards consolidated understanding. Space Sci. Rev. 200, 75. DOI. ADS.

Pant, V., Mazumder, R., Yuan, D., Banerjee, D., Srivastava, A., Shen, Y.: 2016, Simultaneous longitudinal and transverse oscillation in an active filament. Solar Phys. 291. DOI.

Sarkar, S., Pant, V., Srivastava, A.K., Banerjee, D.: 2016, Transverse oscillations in a coronal loop triggered by a jet. Solar Phys. 291. DOI.

Smirnova, V., Konkol, P.M., Solov'ev, A.A., Murawski, K.: 2016, Numerical simulations of solar spicule jets at a magnetic null-point. Solar Phys. 291. DOI. ADS.

Tsap, Y.T., Stepanov, A.V., Kopylova, Y.G.: 2016, Acoustic and slow sausage oscillations in the stratified solar photosphere: hinode observations and phase relationships. Solar Phys. 291. DOI. ADS.

Van Doorsselaere, T., Kupriyanova, E.G., Yuan, D.: 2016, Quasi-periodic pulsations in solar and stellar flares: an overview of recent results (Invited review). Solar Phys. 291. DOI. ADS.

Zaitsev, V.V., Kronshtadtov, P.V., Stepanov, A.V.: 2016, Rayleigh-Taylor instability and excitation of superDreiser electric fields in the solar chromosphere. Solar Phys. 291. DOI. ADS. 